Pacific Journal of Mathematics

EXTREMAL ELEMENTS OF THE CONVEX CONE $B_{n}$ OF 


\title{
EXTREMAL ELEMENTS OF THE CONVEX CONE $B_{n}$ OF FUNCTIONS
}

\author{
E. K. McLachlaN
}

Let $B_{0}$ be the set of nonnegative real continuous on $[0,1]$, let $B_{1}$ be the set of functions belonging to $B_{0}$ such that $\Delta_{h}^{1} f(x)=f(x+h)$ $f(x) \geqq 0, h>0$, for $[x, x+h] \subset[0,1]$, and let $B_{n}, n>1$ be the set of functions belonging to $B_{n-1}$ such that $\Delta_{h}^{n} f(x) \geqq 0$ for $[x, x+n h] \subset[0,1]$ [1]. Since the sum of two functions in $B_{n}$ belongs to $B_{n}$ and since a nonnegative real multiple of a $B_{n}$ function is a $B_{n}$ function, the set of $B_{n}$ functions form a convex cone. It is the purpose of this paper to give the extremal elements [2] of this cone, to prove that they are not dense in a compact convex set that does not contain the origin but meets every ray of the cone, and to show that for the functions of the cone an integral representation in terms of extremal elements is possible. The intersection of the $B_{n}$ cones is the well-known class of functions, the absolutely monotonic functions. Thus the set of these functions form a convex cone also. The extremal elements for this convex cone are given too.

In some correspondence with the author relative to the convex cone $B_{2}$, Professor F. F. Bonsall noted that the extremal elements of $B_{2}$ were the indefinite integrals of the characteristic functions that are extremal elements of the weak closure of $B_{1}$. Professor Bonsall guessed that successive integration would give the extremal elements of $B_{n}$. This proved to be a very good guess, and the author gratefully acknowledges the assistance of these comments.

In the following discussion the vertex of the convex cone is not considered as an extremal element.

1. The convex cone $B_{0}$. For $f \in B_{0}$, then take $f_{1}(x)=x f(x)$ and $f_{2}=f-f_{1}$. Then $f$ is the sum of functions in $B_{0}$ that are not proportional to $f$. Therefore, $B_{0}$ has no extremal elements.

2. The convex cone $B_{1}$. For $f=c>0$ and $f=f_{1}+f_{2}$ where $f_{1}$ and $f_{2} \in B_{1}$ then $0=\Delta_{h}^{1} f(x)=\Delta_{h}^{1} f_{1}(x)+\Delta_{h}^{1} f_{2}(x)$ implies $\Delta_{h}^{1} f_{i}(x)=0$ for $i=1,2$ and $[x, x+h] \subset[0,1]$. Therefore $f_{i}=c_{i}, c_{i}>0, i=1,2$, where $c_{1}+c_{2}=c$. Hence $f$ is an extremal element of $B_{1}$. Now $f=$ $c>0$ belongs also to $B_{n}$ for $n>1$. The set $B_{n}$ is a subcone of $B_{1}$ and hence $f=c$ is again an extremal element of $B_{n}$.

If $f$ is not constant then $f(0)=m$ and $f(1)=M$ and a non-proportional decomposition can be given by taking $f_{1}(x)=\min (f(x),(1 / 2)(M+m))$

Received October 23, 1963. 
and $f_{2}=f-f_{1}$.

3. The convex cone $B_{2}$. The functions of $B_{2}$ are exactly the non-negative, nondecreasing and convex functions on $[0,1][5]$.

Again the positive constant functions are extremal functions. If $f \in B_{2}, f$ is not constant and $f(0)>0$ then take $f_{1}=f(0)$ and $f_{2}=f-f_{1}$. In so doing $f_{1}$ and $f_{2} \in B_{2}$ and $f_{1}$ and $f_{2}$ are not proportional to $f$. Since this same technique still can be used for $B_{n}, n>2$, the only extremal elements of $B_{n}$ such that $f(0)>0$ are the positive constant functions.

If $f(x)=0, x \in[0, \xi]$ and $m(x-\xi)$ for $x \in(\xi, 1]$ where $0 \leqq \xi<1$ and $m>0$, then for $f=f_{1}+f_{2}$ it follows that $f_{1}$ and $f_{2}$ are zero where $f$ is zero and $f_{1}$ and $f_{2}$ are linear where $f$ is linear. Thus $f_{1}$ and $f_{2}$ are proportional to $f$ and $f$ is therefore extremal.

If $f(x)=0, x \in\left[0, \xi_{1}\right], m_{1}\left(x-\xi_{1}\right)$ for $x \in\left(\xi_{1}, \xi_{2}\right], \cdots$,

$$
\sum_{i=1}^{k} m_{i}\left(x-\xi_{i}\right)
$$

for $x \in\left(\xi_{k}, 1\right]$ where $0<\xi_{1}<\xi_{2}<\cdots<\xi_{k}<1$ and $m_{i}>0$ for $i=$ $1,2, \cdots, k$, for $k>1$ then $f \in B_{2}$. Let $f_{1}(x)=0$, for $x \in\left[0, \xi_{1}\right], f_{1}(x)=$ $m_{1}\left(x-\xi_{1}\right)$ for $\left(\xi_{1}, 1\right]$ and $f_{2}=f-f_{1}$. Then $f_{1}$ and $f_{2} \in B_{2}$ and both are not proportional to $f$.

Finally, if $f$ is not any of the above functions, but $f$ belongs to $B_{2}$, let $\xi_{1}=\inf \{x: f(x)>0\}$. Then $0 \leqq \xi_{1}<1$. On $\left[\xi_{1}, 1\right]$, $f$ is convex, $f\left(\xi_{1}\right)=0$ and $f(1)$ is finite. Furthermore, the right-hand derivative at $\xi_{1}, f_{+}^{\prime}\left(\xi_{1}\right)$ is finite and in $\left[\xi_{1}, 1\right] f_{-}^{\prime}$, the left-hand derivative, must take on more than a finite number of values since $f$ is not polygonal on $\left[\xi_{1}, 1\right]$. Thus there exist $\xi_{2}, \xi_{1}<\xi_{2} \leqq 1$ such that on $\left[\xi_{1}, \xi_{2}\right] f_{+}^{\prime}$ is not piecewise linear on three or more non-overlapping segments whose union is $\left[\xi_{1}, \xi_{2}\right]$ and $f_{-}^{\prime}\left(\xi_{2}\right)$ is finite. By Lemma 4 of a paper by the author [4], there exist convex, nonnegative and nondecreasing functions $f_{1}$ and $f_{2}$ different from $f$ on $\left[\xi_{1}, \xi_{2}\right]$ such that $f_{1}$ and $f_{2}$ have the same values and the same derivatives at the end-points as $f$ and $f=\alpha f_{1}+$ $(1-\alpha) f_{2}$ for some $\alpha, 0<\alpha<1$. Thus define $f_{1}$ and $f_{2}$ equal to $f$ on the complement of $\left[\xi_{1}, \xi_{2}\right]$ relative to $[0,1]$ and then $\alpha f_{1}$ and $(1-\alpha) f_{2}$ belong to $B_{2}$ and both are not proportional to $f$.

Thus the extremal elements of $B_{2}$ are positive constant functions and those $f$ such that $f(x)=0, x \in[0, \xi]$ and $f(x)=m(x-\xi)$ for $x \in[\xi, 1]$ where $0 \leqq \xi<1$ and $m>0$. Designate this latter function by $f(\xi, 1 ;)$ for $m=1$.

4. The convex cone $B_{n}, n>2$. The function $f$, such that $f(x)=$ $0, x \in[0, \xi], f(x)=m(x-\xi)^{n-1}, x \in[\xi, 1], 0 \leqq \xi<1$ and $m>0$, that is $m f\left(\xi, n-1\right.$; ) belongs to $B_{n}$ and is an extremal element of $B_{n}$. 
Already $m f\left(\xi, 1\right.$;) belongs to $B_{2}$. Now by induction it shall be shown that $m f(\xi, n-1 ;) \in B_{n}$ for $n>2$. In fact, it is true in general that if $f \in B_{n-1}$ and if

$$
F(x)=\int_{0}^{x} f(t) d t
$$

then $F \in B_{n}^{\cdot} \quad$ For if $\Delta_{h}^{k} f(x) \geqq 0$ for $k=0, \cdots, n-1$ then

$$
\Delta_{h}^{k} F(x)=\Delta_{h}^{k-1} \int_{x}^{x+h} f(t) d t=\Delta_{h}^{k-1} f(\xi)>0
$$

where $x<\xi<x-h$ and $k=0, \cdots, n$. Thus since

$$
m f(\xi, n-1 ; x)=\int_{0}^{x}(n-1) m f(\xi, n-2 ; t) d t
$$

and since by the induction hypothesis $(n-2) m f(\xi, n-2 ;) \in B_{n-1}$, it follows that $m f(\xi, n-1 ;) \in B_{n}$.

Similarly, by induction it shall be shown that $f=m f(\xi,-1$;) is an extremal element of $B_{n}$ It has already been shown that $m f(\xi, 1 ;)$ is an extremal element of $B_{n-1}$ for any $m>0$ and for $0 \leqq \xi<1$. Now let $f=m f(\xi, n-1 ;)=f_{1}+f_{2}$ where $f_{1}$ and $f_{2}$ belong to $B_{n}$. For $n>2$, functions in $B_{n}$ have derivatives, $f_{1}^{\prime}$ and $f_{2}^{\prime}$ on $[0,1$ ) (See [5] Chapter IV) and the functions $f_{1}^{\prime}$ and $f_{2}^{\prime}$ belong to $B_{n-1}$ on $[0, \delta]$ for any $\delta, 0<\delta<1$. Take $\delta<1$ such that $\xi<\delta$, then by the induction hypothesis it follows that $f_{i}^{\prime}$ and $f_{2}^{\prime}$ are proportional to $f^{\prime}=(n-1) m$ $f(\xi, n-2 ;)$ on $[0, \delta]$. Hence $f_{i}(x)=\lambda_{i} f(x)+c_{i}, x \in[0, \delta], 0 \leqq \lambda_{i}$, where $c_{i}$ is a constant for $i=1,2$. Since $f_{1}(0)=f_{2}(0)=(n-1) m$ $f(\xi, n-2 ; 0)=0$ it follows that $c_{i}=0, i=1,2$ and hence $f_{1}$ and $f_{2}$ are proportional to $f$ on $[0, \delta]$ for any $\delta, 0<\delta<1$. However, since $f, f_{1}$ and $f_{2}$ are continuous on $[0,1]$, it follows then that $f_{1}$ and $f_{2}$ are proportional to $f$ on $[0,1]$. Therefore, $m f(\xi, n-1$;) is an extremal element of $B_{n}$.

Notice that like the positive constant functions these functions $m f(\xi, n-1$;) for $\xi=0$, that is the functions $m f(0, n-1$;) belong to $B_{n}$ for all $n$ since its derivatives of all orders exist and are nonnegative on $[0,1]$. However, if $\xi>0$, let $s$ and $k$ be integers such that $s>k$ and let $x$ and $h$ be such that $x+(s-2) h=\xi, 0 \leqq x<x+s h \leqq 1$. Then

$$
\Delta_{h}^{s} m f(\xi, k ; x)=m\left[(2 h)^{k}-s(h)^{k}\right]=m h^{k}\left(2^{k}-s\right) .
$$

Hence, if $s>2^{k}$, then the expression on the right is negative and thus $m f(\xi, k ;) \notin B_{s}$. This means that whereas $m f(\xi, n-1 ;) \in B_{n}$ it does not belong to $B_{j}$ for $j>2^{n-1}$.

It remains only to show that the functions of $B_{n}$ other than the 
positive constant functions of the form $m f(\xi, k ;), 0 \leqq \xi<1, m>0$, $k=1,2, \cdots, n-1$ that belong to $B_{n}$ are not extremal elements of $B_{n}$. It is known that $f^{\prime}$ exists and is a continuous function on $[0,1)$. If $f^{\prime}$ can be extended to be a continuous function on $[0,1]$, that is, if $\lim f^{\prime}(x)$ as $x \rightarrow 1^{-}$exists and is finite, then $f^{\prime} \in B_{n-1}$. By assuming the induction hypothesis on $n$, there exist functions $g_{1}$ and $g_{2}$ belonging to $B_{n-1}$ such that $f^{\prime}=g_{1}+g_{2}$ and $g_{1}$ and $g_{2}$ are not proportional to $f^{\prime}$. Let $f_{i}(x)=\int_{0}^{x} g_{i}(t) d t, i=1,2$. Thus $f_{1}$ and $f_{2}$ belong to $B_{n}$ and they are not proportional to $f$. For if $f_{1}=\lambda_{1} f, \lambda_{1} \geqq 0$, then $f_{1}^{\prime}=\lambda_{1} f^{\prime}=g_{1}$. This clearly violates what is known about $g_{1}$. Hence such a function $f$ is not an extremal element of $B_{n}$.

Finally, suppose that $f \in B_{n}$ and $\lim f^{\prime}(x)=+\infty$ as $x \rightarrow 1^{-}$. Then the following must be true: $f^{\prime}, f^{\prime \prime}, \cdots, f^{(n-2)}$ and $f_{+}^{(n-1)}$, the right-hand derivative of $f^{(n-2)}$ are defined on $[0,1)$; each of them approaches $+\infty$ as $x$ approaches one from the left; and $\Delta_{h}^{k} f^{(j)}(x) \geqq 0$ for $0 \leqq x<1$, $j=1,2, \cdots, n-1$, (with the special understanding for $j=n-1$ ), $k=0,1,2, \cdots, n-j$. Denote by $B_{n-j}[0,1)$ the set of real functions $\phi$ of $[0,1) \Delta_{h}^{k} \phi(x) \geqq 0,0 \leqq x<1, k=0,1, \cdots, n-j$ for $j=1,2, \cdots$, $n-1$ such that $\phi(x) \rightarrow+\infty$ as $x \rightarrow 1^{-}$. The functions $B_{n-j}[0,1)$ form a convex cone and $f^{(j)} \in B_{n-j}[0,1)$ for $j=1, \cdots, n-1$. By an argument similar to the one given earlier, the indefinite integral of a function $F$ in $B_{m}[0,1)$ belongs to $B_{m+1}[0,1)$ if $\int_{0}^{x} F(t) d t \rightarrow+\infty$ as $x \rightarrow 1^{-}$. Also if $g, g_{1}$ and $g_{2} \in B_{m}[0,1), g=g_{1}+g_{2}$, and $g_{1}$ and $g_{2}$ are not proportional to $g$, then the indefinite integrals of $g_{1}$ and $g_{2}$ are not proportional to $g$. Not that if $g=g_{1}+g_{2}$ as above and if $\int_{0}^{1-} S(t) d t$ is finite, then the same will be true of $\int_{0}^{1-} g_{i}(t) d t$ for $i=1,2$. If the $\lim g(t)=+\infty$ as $t \rightarrow 1^{-}$and $\int_{0}^{1-} g_{i}(t) d t=+\infty$ then the same will be true of $\int_{0}^{1-} g_{i}(t) d t$ for $i=1,2$ if there exists constants $\gamma_{i}>0, i=1,2$ such that $g_{i}(t) \geqq$ $\gamma_{i} g(t)$ for some $\delta, 0<\delta<1$. For the case when $\int_{0}^{1-} g_{i}(t) d t$ is finite then $f_{i}$ where $f_{i}(x)=\int_{0}^{x} g_{i}(t) d t, i=1,2$ can be extended into a function that is continuous on $[0,1]$. Hence $f_{1}$ and $f_{2}$ will belong to $B_{m+1}$.

Thus the object is to find two functions $g_{1}$ and $g_{2}$ that belong to $B_{1}[0,1)$, such that $f_{+}^{(n-1)}=g_{1}+g_{2}, g_{1}$ and $g_{2}$ are not proportional to $f_{+}^{(n-1)}$, and such that $g_{i}(t) \geqq \lambda_{i} f_{\perp-1)}^{(n-1)}(t), \delta \leqq t<1, \delta>0$. Then $f_{1}$ given by

$$
f_{i}(x)=\int_{0}^{x} \int_{0}^{t_{n-2}} \cdots \int_{0}^{t_{2}} \int_{0}^{t_{1}} g_{i}(t) d t d t_{1} \cdots d t_{n-2},
$$

$i=1,2$ belong to $B_{n}$ and give a nonproportional decomposition of $f$. The lemma below shows how the functions $g_{1}$ and $g_{2}$ with the desired properties can be constructed. 
LEMma. Given $f$ on $[0,1)$ such that $f$ is right continuous, nonneqative, nondecreasing and $f(x) \rightarrow+\infty$ as $x \rightarrow 1$. There exist two functions $f_{1}$ and $f_{2}$ on $[0,1)$ that are right continuous, nonnegative and nondecreasing, $f=f_{1}+f_{2}, f_{1}$ and $f_{2}$ are not proportional to $f$, and $f_{i}(x) \geqq \gamma_{i} f(x)$ on $[\delta, 1)$ for some $0<\delta<1$ and $\gamma_{i}>0, i=1,2$.

Proof. All the discontinuities of $f$ must be jump discontinuities. If the point $x=1$ is an accumulation point of the discontinuities of $f$, then there exist $c_{1}, c_{2}$ and $c_{3}, 0<c_{1}<c_{2}<c_{3}<1$ such that $f$ has a jump of $\theta_{i}$ at $c_{i}, \theta_{i}>0, i=1,2,3$. Take $\theta=(1 / 2) \min \left(\theta_{1} \theta_{2} \theta_{3}\right)$. Let $f_{1}$ be such that $f_{1}(x)=(1 / 2)(f(x)-\theta), c_{1} \leqq x<c_{2}, f_{1}(x)=(1 / 2)(f(x)+\theta)$, $c_{2} \leqq x<c_{3}$ and $f_{1}(x)=(1 / 2) f(x)$ otherwise. Take $f_{2}=f-f_{1}$. Then $f_{1}$ and $f_{2}$ have the required properties.

If the point $x=1$ is not an accumulation point of the discontinuities then there exists $\delta, 0<\delta<1$ such that $f$ is continuous on $[\delta, 1)$. Let $\xi$ be a point such that $f(\xi)=f(\delta)+1$, then $\delta \leqq \xi<1$. Take $f_{1}$ such that $f_{1}(x)=(1 / 2) f(x), 0 \leqq x<\xi$ and $f_{1}(x)=(1 / 3)(f(x)-f(\delta)-1)+$ $(1 / 2)(f(\delta)+1), \xi \leqq x<1$. Let $f_{2}=f-f_{1}$. Then again $f_{1}$ and $f_{2}$ have the required properties.

5. Absolutely monotonic functions. The continuous functions $f$ on $[0,1]$ such that $f^{(k)}(x) \geqq 0$ for $0<x<1, k=0,1,2, \cdots$ were called absolutely monotonic functions by Bernstein. These functions clearly form a convex cone of functions on $[0,1]$. Since the functions $f$ belonging to $B_{n}, n>2$, have $f^{(k)}(x) \geqq 0, k=0,1, \cdots, n-2$, it follows that $\bigcap_{n=0}^{\infty} B_{n}$ is contained in the set of absolutely monotonic functions. Since the continuous functions $f$ on $[0,1]$ such that $f^{(k)}(x) \geqq 0, k \leqq n$ on $(0,1)$ have $A_{h}^{k} f(x) \geqq 0$ for $k \leqq n$, then $\bigcap_{n=0}^{\infty} B_{n}$ is the set of absolutely monotonic functions. Denote this set by $B_{\infty}$

From the earlier remarks it is clear that $c_{0}, c_{1} x, c_{2} x^{2}, \cdots$ belong to $B_{\infty}$ for $c_{i}>0, i=0,1,2, \cdots$ and they are indeed extremal elements of $B_{\infty}$. Since any $f \in B_{\infty}$ is absolutely monotonic on $[0,1)$ it follows that

$$
f(x)=\sum_{n=0}^{\infty} f^{(n)}(0)\left(x^{n} / n !\right), \quad 0 \leqq x<1 .
$$

Consequently, if as many as two terms are nonzero in the series expansion, then take $f_{1}$ equal to one of the two nonzero terms and $f_{2}=f-f_{1}$. Then clearly $f_{1}$ and $f_{2}$ belong to $B_{\infty}$ and $f$ has a nonproportional decomposition. Hence the only extremal elements of $B_{\infty}$ are the functions $c_{i} x^{i}, c_{i}>0, i=0,1,2, \cdots$.

The following theorem summarizes all of the results up to this point.

THEOREM. The convex cone $B_{0}$ has no extremal elements. The 
functions $f=c>0$, where $c$ is a constant, are extremal elements of $B_{n}, n=1,2,3, \cdots$. The function $m f(\xi, n-1 ; x)=0$ for $0 \leqq x<\xi$ and $m(x-\xi)^{n-1}$ for $\xi \leqq x \leqq 1, m>0,0 \leqq \xi<1$ are extremal elements of $B_{n}, n=2,3, \cdots$. The only other extremal elements of $B_{n}, n=$ $2,3, \cdots$ are those functions $m f(\xi, k ;), k=1,2, \cdots, n-2$ that belong to $B_{n}$. The extremal elements of the convex cone $B_{\infty}$, the absolutely monotonic functions, are the functions of the form $c_{i} x^{i}, c_{i}>0, i=$ $0,1,2, \cdots$.

6. Integral representations. The set of functions $B_{n}-B_{n}, n \geqq$ 1 , form a linear space containing the convex cone $B_{n}$. Using the topology of simple convergence $B_{n}-B_{n}$ becomes a locally convex space. Let $C_{n}$ be the set of functions $f$ of $B_{n}$ such that $f(1)=1$. Clearly, $C_{n}$ meets every ray of $C_{n}$ once and only once and does not meet the origin in $B_{n}-B_{n}$, that is the zero function. Furthermore, $C_{n}$ is convex. Each function $f$ of $C_{n}$ is such that $0 \leqq f(x) \leqq 1$ for all $0 \leqq x \leqq 1$ since $f$ is nonnegative and nondecreasing. It follows by use of the Tychonoff theorem that $C_{n}$ is contained in a compact set in $B_{n}-B_{n}$, namely $\left\{f: f \in B_{n}-B_{n}, 0 \leqq f(x) \leqq 1,0 \leqq x \leqq 1\right\}$. Thus $C_{n}$ is compact, if it can be shown that $C_{n}$ is closed. This will be done by showing the complement of $C_{n}$ is open.

If $g \in B_{n} \backslash C_{n}$ then $g(1) \neq 1$. The set

$$
V(1 ; \varepsilon)+g=\left\{f: f \in B_{n}-B_{n},|f(1)-g(1)|<\varepsilon\right\}
$$

where $\varepsilon=(1 / 2)|1-g(1)|$ is an open set about $g$ that fails to meet $C_{n}$. If $g \notin B_{n}$ then there exists $x_{0}, k$ and $h$ such that $\Delta_{h}^{k} g\left(x_{0}\right)=\delta<0$. Now

$$
\Delta_{h}^{k} g\left(x_{0}\right)=\sum_{j=0}^{k}(-1)^{j}\left(\begin{array}{l}
k \\
j
\end{array}\right) g\left(x_{0}+(k-j) h\right) .
$$

Consider

$$
\begin{aligned}
& V=V\left(x_{0}, x_{0}+h, \cdots, x_{0}+k h ; \varepsilon\right)+g \\
& =\left\{f: f \in B_{n}-B_{n},\left|f\left(x_{0}+j h\right)-g\left(x_{0}+j h\right)\right|<\varepsilon, j=0,1, \cdots, k\right\} .
\end{aligned}
$$

where $\varepsilon=2^{-(k+1)}(-\delta)$. Then $V$ does not meet $C_{n}$ since for if $f \in V$

$$
\begin{aligned}
\Delta_{h}^{k} f\left(x_{0}\right) & =\Delta_{h}^{k}\left(f\left(x_{0}\right)-g\left(x_{0}\right)\right)+\Delta_{h}^{k} g\left(x_{0}\right) \\
& <\left|\Delta_{h}^{k}\left(f\left(x_{0}\right)-g\left(x_{0}\right)\right)\right|+\Delta_{h}^{k} g\left(x_{0}\right) \\
& <\sum_{j=0}^{k}\left(\begin{array}{l}
k \\
j
\end{array}\right)\left|f\left(x_{0}+(k-j) h\right)-g\left(x_{0}+(k-j) h\right)\right|+\delta \\
& <\varepsilon \sum_{j=0}^{k}\left(\begin{array}{l}
k \\
j
\end{array}\right)+\delta \\
& =\varepsilon 2^{k}+\delta \\
& =(1 / 2) \delta<0 .
\end{aligned}
$$


Hence $f \notin B_{n}$.

Thus by Theorem 39.4 of Choquet [3], it follows that for any function $f_{0}$ in $C_{n}$ there exists a nonnegative measure $\mu_{0}$ on the closure of the extreme points of $C_{n}$ such that $f_{0}(x) \int d \mu_{0}=\int f(x) d \mu_{0}$. Since $C_{n}$ meets every ray of the cone $B_{n}$ and does not contain the origin, it follows that each function of $B_{n}$ is a scalar multiple of such a representation.

If the set of extremal elements of $C_{n}$ are dense in $C_{n}$, then the above result would be of no interest, but this is not the case. Consider $g_{0}(x)=(1 / 2)+2^{n-2} f(1 / 2, n-1 ; x)$. Then $g_{0}$ belongs to $B_{n}$ since it is the sum of two functions in $B_{n}$. Notice further that $g_{0}(1)=1$ and hence $g_{0} \in C_{n}$. The neighborhood of $g_{0}$,

$$
\begin{aligned}
V_{0} & =V(0,1 ; 1 / 8)+g_{0} \\
& =\left\{f: f \in B_{n}-B_{n},\left|f(i)-q_{0}(i)\right|<(1 / 8), i=0,1\right\},
\end{aligned}
$$

does not meet any extreme point of $C_{n}$. Any positive constant function of $C_{n}$ is $f(x)=1$ for all $x$ and hence $f(0)>5 / 8$ at $x=0$. Any function of the form $m f(\xi, k$; ) that belongs to zero at $x=0$ and hence does not belong to $V_{0}$.

7. Remarks. Choquet [3] discusses convex cones of functions related to the cones discussed here. The main difference is that the differences, $\Delta_{h}^{k} f(x)$, alternate in sign as $k$ takes on successive integral values in the cones that Choquet considered.

\section{REFERENCES}

1. F. F. Bonsall, Semi-alqebras of continuous functions, Proceedings of the International Symposium on Linear Spaces, (1961), 101-114.

2. N. Bourbaki, Espaces vectoriels topologiques, Act. Sci. Ind. no. 1189, Paris, 1953.

3. G. Choquet, Theory of capacities, Annales de 1'Institut Fourier, 5 (1953 and 1954), 131-256.

4. E. K. McLachlan, Extremal elements of the convex cone of semi-norms, Pacifie J. Math., 13 (1963), 1335-1342.

5. D. V. Widder, The Laplace transform, Princeton Mathematics Series, 6 (1946).

OKLAHoma State University 



\section{PACIFIC JOURNAL OF MATHEMATICS}

\section{EDITORS}

\author{
Robert Osserman \\ Stanford University \\ Stanford, California
M. G. Arsove
University of Washington
Seattle 5 , Washington

\author{
J. DugundJI \\ University of Southern California \\ Los Angeles 7, California
}

Lowell J. Paige

University of California

Los Angeles 24, California

\section{ASSOCIATE EDITORS}
E. F. BECKENBACH
B. H. NeumanN
F. WOLF
K. YOSIDA

\section{SUPPORTING INSTITUTIONS}

\author{
UNIVERSITY OF BRITISH COLUMBIA \\ CALIFORNIA INSTITUTE OF TECHNOLOGY \\ UNIVERSITY OF CALIFORNIA \\ MONTANA STATE UNIVERSITY \\ UNIVERSITY OF NEVADA \\ NEW MEXICO STATE UNIVERSITY \\ OREGON STATE UNIVERSITY \\ UNIVERSITY OF OREGON \\ OSAKA UNIVERSITY \\ UNIVERSITY OF SOUTHERN CALIFORNIA
}

\author{
STANFORD UNIVERSITY \\ UNIVERSITY OF TOKYO \\ UNIVERSITY OF UTAH \\ WASHINGTON STATE UNIVERSITY \\ UNIVERSITY OF WASHINGTON \\ * * * * \\ AMERICAN MATHEMATICAL SOCIETY \\ CALIFORNIA RESEARCH CORPORATION \\ SPACE TECHNOLOGY LABORATORIES \\ NAVAL ORDNANCE TEST STATION
}

Mathematical papers intended for publication in the Pacific Journal of Mathematics should by typewritten (double spaced), and on submission, must be accompanied by a separate author's résumé. Manuscripts may be sent to any one of the four editors. All other communications to the editors should be addressed to the managing editor, L. J. Paige at the University of California, Los Angeles 24, California.

50 reprints per author of each article are furnished free of charge; additional copies may be obtained at cost in multiples of 50 .

The Pacific Journal of Mathematics is published quarterly, in March, June, September, and December. Effective with Volume 13 the price per volume (4 numbers) is $\$ 18.00$; single issues, $\$ 5.00$. Special price for current issues to individual faculty members of supporting institutions and to individual members of the American Mathematical Society: $\$ 8.00$ per volume; single issues $\$ 2.50$. Back numbers are available.

Subscriptions, orders for back numbers, and changes of address should be sent to Pacific Journal of Mathematics, 103 Highland Boulevard, Berkeley 8, California.

Printed at Kokusai Bunken Insatsusha (International Academic Printing Co., Ltd.), No. 6, 2-chome, Fujimi-cho, Chiyoda-ku, Tokyo, Japan.

PUBLISHED BY PACIFIC JOURNAL OF MATHEMATICS, A NON-PROFIT CORPORATION

The Supporting Institutions listed above contribute to the cost of publication of this Journal, but they are not owners or publishers and have no responsibility for its content or policies. 


\section{Pacific Journal of Mathematics}

\section{Vol. 14, No. $3 \quad$ July, 1964}

Erik Balslev and Theodore William Gamelin, The essential spectrum of a class of ordinary differential operators . . . . . . . . . . . . . . . . . . . .

James Henry Bramble and Lawrence Edward Payne, Bounds for derivatives in

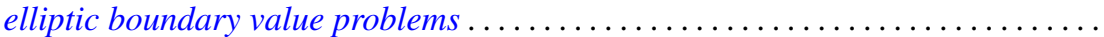

Hugh D. Brunk, Integral inequalities for functions with nondecreasing

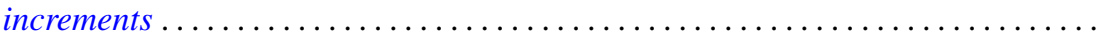

William Edward Christilles, A result concerning integral binary quadratic

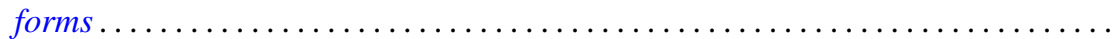

Peter Crawley and Bjarni Jónsson, Refinements for infinite direct decompositions of

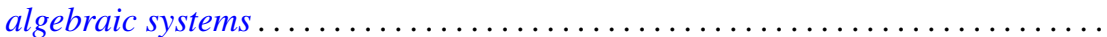

Don Deckard and Carl Mark Pearcy, On continuous matrix-valued functions on a Stonian space.

Raymond Frank Dickman, Leonard Rubin and P. M. Swingle, Another

characterization of the $n$-sphere and related results $\ldots \ldots \ldots \ldots \ldots \ldots$

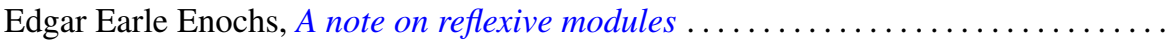

Vladimir Filippenko, On the reflection of harmonic functions and of solutions of the

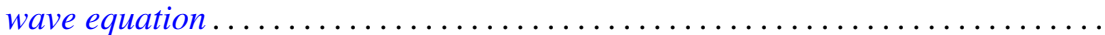

Derek Joseph Haggard Fuller, Mappings of bounded characteristic into arbitrary

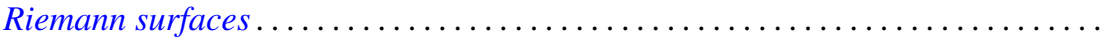
895

Curtis M. Fulton, Clifford vectors . . . . . . . . . . . . . . . . . . . . . . . . . . . . . . 917

Irving Leonard Glicksberg, Maximal algebras and a theorem of Radó . .

919

Kyong Taik Hahn, Minimum problems of Plateau type in the Bergman metric

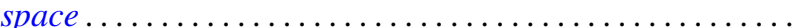

A. Hayes, A representation theory for a class of partially ordered rings...

J. M. C. Joshi, On a generalized Stieltjes trasform

J. M. C. Joshi, Inversion and representation theorems for a generalized Laplace transform ...

Eugene Kay McLachlan, Extremal elements of the convex cone $B_{n}$ of functions ...

Robert Alan Melter, Contributions to Boolean geometry of p-rings ...

James Ronald Retherford, Basic sequences and the Paley-Wiener criterion . . . . . . . 1019

Dallas W. Sasser, Quasi-positive operators. .

Oved Shisha, On the structure of infrapolynomials with prescribed coefficients ..

Oved Shisha and Gerald Thomas Cargo, On comparable means

Maurice Sion, A characterization of weak ${ }^{*}$ convergence ........

Morton Lincoln Slater and Robert James Thompson, A permanent inequality for

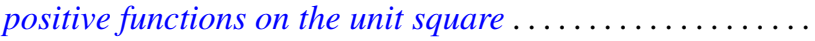

David A. Smith, On fixed points of automorphisms of classical Lie algebras ...

Sherman K. Stein, Homogeneous quasigroups ................

J. L. Walsh and Oved Shisha, On the location of the zeros of some infrapolynomials with prescribed coefficients .

Ronson Joseph Warne, Homomorphisms of $d$-simple inverse semigroups with identity . 